\title{
Robust Stability of Iterative Learning Control Schemes
}

\author{
Mark French* \\ International Journal of Robust and Nonlinear Control \\ To appear
}

\begin{abstract}
A notion of robust stability is developed for iterative learning control in the context of disturbance attenuation. The size of the unmodelled dynamics is captured via a gap distance, which in turn is related to the standard $\mathcal{H}^{2}$ gap metric, and the resulting robustness certificate is qualitatively equivalent to that obtained in classical robust $\mathcal{H}^{\infty}$ theory. A bound on the robust stability margin for a specific adaptive ILC design is established.
\end{abstract}

\section{Introduction}

The field of iterative learning control (known as ILC) is concerned with procedures for learning control tasks (typically in the context of high precision tracking) over a number of 'learning trials' of finite time duration, whereby the process is reset between trials, and the controller is altered from trial to trial to improve performance. This area was motivated originally in the context of robotics [1], and has subsequently developed a more abstract footing in general control theory, and applications in a variety of domains have been considered: see for example the surveys [11], [12], and see [2], [3] for representative recent application examples. It is pertinent to observe that both iterative learning control and the related area of repetitive control has achieved considerable success in applications, perhaps in contrast to the other major theory of learning in control, namely adaptive control.

Underpinning the practical success of any control design, is the requirement for robustness. It is surprising therefore that there is little theoretical understanding of robustness of iterative learning schemes, particularly considering their successful application strongly suggests inherent robustness properties. This should be tempered by the fact that in many ILC schemes 'long term stability' problems have been observed in practical implementations and simulations, see e.g. [9]. This phenomena results in divergence of the error profile after a large number of trials including a period of apparent convergence, and has been attributed variously to the effects of disturbances, numerical errors, and/or un-modelled dynamics. Indeed, to the best knowledge of the author, studies of robustness of ILC are limited to plants with multiplicative perturbations. It is well known that in general multiplicative perturbations alone do not capture all the features of closed loop uncertainties, for example, such perturbations can never describe the difference between an open loop stable and an open loop unstable plant, and such differences are not

\footnotetext{
${ }^{*}$ School of Electronics and Computer Science, University of Southampton, SO17 1BJ, UK, mcf@ecs.soton.ac.uk
} 
necessarily significant for control (see e.g. [15]). In particular, [8], asserts robustness to positive real multiplicative uncertainties (limited by $\pm 90^{\circ}$ phase variations over all frequencies) and [14] and [10] also consider multiplicative perturbations, and relate robust stability of an underlying feedback controller to the related ILC algorithm.

These earlier results are of great interest, but the conditions are too strong for practical use. The aim of this paper is to demonstrate that for a class of ILC designs, provable robustness properties hold. In particular, the class of uncertainties permitted are the classical co-prime factor uncertainty structures studied in robust linear control, and the size of the robustness margin is computed.

In the context of linear systems, the classical robust stability margin $b_{P, C}$ is defined to be the maximum radius of a ball centred on $P$ in the $\mathcal{H}^{2}$ gap metric for which the controller $C$ is guaranteed to stabilize all plants $P_{1}$ within [16], [15]. The standard $\mathcal{H}^{2}$ gap $\delta\left(P, P_{1}\right)$ measures the size of the smallest stable co-prime factor perturbation between normalised co-prime factor representations of a pair of plants $P$ and $P_{1}$. This paper develops a similar framework for the analysis of iterative learning schemes, and constructs a robustness margin for a particular ILC design.

In particular, we consider the classical class of relative degree one linear systems with known high frequency gain, coupled with an adaptive ILC scheme (see e.g. [6], [13]). The control task is stabilization, i.e. tracking of the zero output trajectory, and is to be achieved in the face of $L^{p}$ input and output disturbances acting over all the trials. The resulting robust stability margin $B_{P, C}(r)$ is shown to be strictly positive, and dependent on the size of the disturbances $r>0$. That is, the objective of tracking zero can be realized asymptotically for all plants within the robust stability margin. The resulting margin can be interpreted relative to the standard $\mathcal{H}^{2}$ gap [16], [15].

At present, the analysis is restricted to stabilization problems, namely the tracking of the zero trajectory. Conventional ILC designs aim to achieve high precision tracking of more general trajectories. ${ }^{1}$ The extension of the current robustness results to tracking are the topic of current research; however, we observe that we note three reasons for the interest in the stabilization specifications considered:

1. Tracking of the zero trajectory is a particular case of the general tracking problem, therefore it is necessary that good robustness results can be achieved in this special case as a precursor to more general tracking results.

2. The problem studied is actually that of disturbance attenuation: stabilization has to be achieved in the presence of input and output disturbances and gain function bounds are given; this a legitimate ILC problem in its own right.

3. The analogous adaptive control problem has been extensively studied, and gap robustness results are available [4],[5]. A comparison of the nature of robust stability margin between the adaptive and the ILC settings shows the importance of the stabilizing effect of reinitializing the process at the start of every ILC trial. In the adaptive problem, the state maintains a memory of the past which builds up and makes a large contribution to reducing the size of the robustness margin.

\footnotetext{
${ }^{1}$ The ILC designs considered also have tracking properties (when the output disturbance is taken to be the reference), but we do not consider this point further in this paper.
} 
Thus we will establish provable conditions under which an iterative learning scheme works with no long term stability problems in the presence of a realistic class of un-modelled dynamics and disturbances.

\section{Statement of the robust iterative learning problem and main result}

We let $\mathcal{U}, \mathcal{Y}$ denote normed signal spaces corresponding respectively to the input and output signals. Specifically, all signal spaces considered are taken to be $\mathbb{R}$ valued $L^{p}[\mathcal{T}]$ spaces defined over the one of the following three domains: $\mathcal{T}=[0, T], \mathbb{R}_{+}, \mathbb{N} \times[0, T]$. The first two domains have a natural total ordering, whilst the two-dimensional domain is totally ordered as follows: $\left(k_{1}, t_{1}\right)=\tau_{1}<\tau_{2}=\left(k_{2}, t_{2}\right)$ if and only if $k_{1}<k_{2}$ or if $k_{1}=k_{2}$ and $t_{1}<t_{2}$. A truncation operator is defined as follows:

$$
T_{\tau}(v)= \begin{cases}v(t), & t<\tau \\ 0, & t \geq \tau\end{cases}
$$

The plant and controller operators $\Sigma, \Xi$ will be assumed to be causal, in the sense that if $T_{\tau} u=T_{\tau} v$ then $T_{\tau} \Sigma u=T_{\tau} \Sigma v$ and $T_{\tau} \Xi u=T_{\tau} \Xi v$. Given a signal space $\mathcal{U}$ (resp. $\mathcal{Y}$ ), the extended space $\mathcal{U}_{e}\left(\right.$ resp. $\left.\mathcal{Y}_{e}\right)$, is defined

$$
\mathcal{U}_{e}=\left\{u \in \operatorname{map}(\mathcal{T}, \mathbb{R}) \mid\left\|T_{\tau} u\right\|_{\mathcal{U}}<\infty \forall \tau>0\right\},
$$

where $\operatorname{map}(\mathcal{T}, \mathbb{R})$ denotes the set of all functions $\mathcal{T} \rightarrow \mathbb{R}$.

Throughout this paper we consider feedback loop $[\Sigma, \Xi]$ given by the equations:

$$
[\Sigma, \Xi] \quad: \quad \begin{array}{ll}
y_{1}=\Sigma u_{1}, & y_{0}=y_{1}+y_{2} \\
& u_{2}=\Xi y_{2},
\end{array}
$$

where $u_{0}, u_{1}, u_{2} \in \mathcal{U}_{e}, y_{0}, y_{1}, y_{2} \in \mathcal{Y}_{e}$ and $\Sigma: \mathcal{U}_{e} \rightarrow \mathcal{Y}_{e}, \Xi: \mathcal{Y}_{e} \rightarrow \mathcal{U}_{e}$ for appropriate signal spaces $\mathcal{U}, \mathcal{Y}$, see figure 1 . Here $\left(u_{0}, y_{0}\right) \in \mathcal{W}_{e}$, (where $\mathcal{W}=\mathcal{U} \times \mathcal{Y}$ ), are the external signals (or disturbances), and $u_{1}, u_{2}, y_{1}, y_{2}$ are the internal signals.

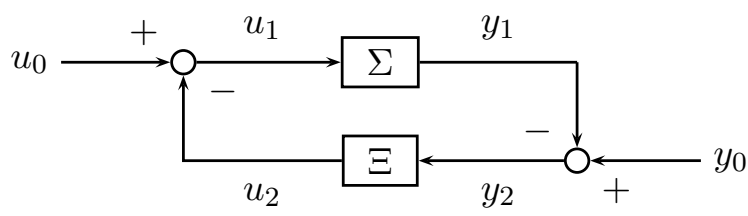

Figure 1: The closed-loop system $[\Sigma, \Xi]$.

It is implicit in this definition that the feedback loop is globally well posed, namely that if the external signals $\left(u_{0}, y_{0}\right)$ lie in the extended space $\mathcal{W}_{e}$, then the internal signals are also locally bounded, that is $u_{1}, u_{2} \in \mathcal{U}_{e}, y_{1}, y_{2} \in \mathcal{Y}_{e}$. Equivalently, the following operator is defined:

$$
\Pi_{\Sigma, \Xi}: \mathcal{W}_{e} \rightarrow \mathcal{W}_{e} \quad: \quad\left(\begin{array}{c}
u_{0} \\
y_{0}
\end{array}\right) \mapsto\left(\begin{array}{c}
u_{1} \\
y_{1}
\end{array}\right)
$$


since it is straightforward to observe that if $u_{0}, u_{1} \in \mathcal{U}_{e}, y_{0}, y_{1} \in \mathcal{Y}_{e}$ then $u_{2} \in \mathcal{U}_{e}, y_{2} \in \mathcal{Y}_{e}$. Note that this global well-posedness property (which is equivalent to excluding finite escape times), will be verified for all the concrete cases considered later in the paper.

The closed loop $[\Sigma, \Xi]$ is said to be stable if $\Pi_{\Sigma, \Xi}(\mathcal{W}) \subset \mathcal{W}$, that is that bounded external signals implies bounded internal plant signals $u_{1} \in \mathcal{U}, y_{1} \in \mathcal{Y}$ (and consequently bounded internal controller signals $u_{2} \in \mathcal{U}, y_{2} \in \mathcal{Y}$, similarly to the previous observation).

The graph of an plant operator $\Sigma: \mathcal{U}_{e} \rightarrow \mathcal{Y}_{e}$ is the collection of all bounded input, output pairs compatible with the plant equations:

$$
\mathcal{G}_{\Sigma}^{\mathcal{W}}=\left\{\left(u_{1}, y_{1}\right)^{T} \in \mathcal{U}_{e} \times \mathcal{Y}_{e}: y_{1}=\Sigma u_{1}, u_{1} \in \mathcal{U}, y_{1} \in \mathcal{Y}\right\}
$$

Similarly, the controller operator graph is defined as follows:

$$
\mathcal{G}_{\Xi}^{\mathcal{W}}=\left\{\left(u_{2}, y_{2}\right)^{T} \in \mathcal{U}_{e} \times \mathcal{Y}_{e}: u_{2}=\Xi y_{2}, u_{2} \in \mathcal{U}, y_{2} \in \mathcal{Y}\right\}
$$

Stability of $[\Sigma, \Xi]$ is then equivalent to the relation $\mathcal{W}=\mathcal{G}_{\Sigma}^{\mathcal{W}} \oplus \mathcal{G}_{\Xi}^{\mathcal{W}}$. Finally we observe that if $\Sigma$ is stabilizable in the sense that for all $u \in L^{p}[0, T]$ there exists $v \in L^{p}\left[\mathbb{R}_{+}\right]$such that $\left.v\right|_{[0, T]}=u$ and $\left.(v, \Sigma v)^{T} \in \mathcal{G}_{\Sigma}^{L^{p}} \mathbb{R}_{+}\right]$, then

$$
\mathcal{G}_{\Sigma}^{L^{p}[0, T]}=\left.\mathcal{G}_{\Sigma}^{L^{p}\left[\mathbb{R}_{+}\right]}\right|_{[0, T]}
$$

\subsection{Plant dynamics along the trial}

We first consider the case where $\mathcal{U}=L^{p}[0, T], \mathcal{Y}=L^{p}[0, T]$. The nominal plant

$$
\Sigma: L_{e}^{p}[0, T] \rightarrow L_{e}^{p}[0, T]
$$

is assumed to be linear, time invariant, single input, single output, relative degree one and where the sign of the high frequency gain $b$ is known. Such a plant can be expressed in the following form:

$$
\begin{aligned}
\frac{d}{d t}\left(\begin{array}{c}
y_{1} \\
z
\end{array}\right) & =\left[\begin{array}{cc}
-a_{11} & a_{12} \\
a_{21} & A_{22}
\end{array}\right]\left(\begin{array}{c}
y_{1} \\
z
\end{array}\right)+\left(\begin{array}{l}
b \\
0
\end{array}\right) u_{1} \quad x(0)=\left[\begin{array}{c}
y_{1}(0) \\
z(0)
\end{array}\right]=0 . \\
\Sigma\left(u_{1}\right) & =y_{1}
\end{aligned}
$$

where $a_{11} \in \mathbb{R}, a_{12}, a_{21} \in \mathbb{R}^{n-1}, A_{22} \in \mathbb{R}^{(n-1) \times(n-1)}$, and without loss of generality $b=1 \in \mathbb{R}$. The following property for $\Sigma$ is critical to the analysis that follows, namely that there exists a constant $M_{T}>0$ such that

$$
\left\|a_{12} T_{T} z\right\| \leq M_{T}\left\|T_{T} y_{1}\right\| .
$$

The set of all such plants is denoted by $\mathcal{M}_{M_{T}}^{1}$. Note that if $\Sigma$ is minimum phase then $M$ can be chosen to be independent of $T>0$.

A second plant operator $\Sigma_{1}: L_{e}^{p}[0, T] \rightarrow L_{e}^{p}[0, T]$ will also be considered. This represents the 'actual' plant (thus containing the unmodelled dynamics), and is assumed to be linear, time invariant and single input, single output. 


\subsection{Dynamics over the trials}

The iterative learning problem consists of applying the control repeatedly over a number of trials, each of length $T$, with the aim of improving performance as the trials progress. The signal spaces are taken to be $\mathcal{U}=\mathcal{Y}=L^{p}(\mathbb{N} \times[0, T])$, with norm:

$$
\|x(\cdot, \cdot)\|=\left(\sum_{i=1}^{\infty}\|x(i, \cdot)\|_{L^{p}[0, T]}^{p}\right)^{\frac{1}{p}} .
$$

The first argument is interpreted as the trial index, the second the time elapsed along the trial. The nominal and actual plants are unchanged between the trials:

$$
\begin{array}{clll}
P_{\Sigma}: L_{e}^{p}(\mathbb{N} \times[0, T]) \rightarrow L_{e}^{p}(\mathbb{N} \times[0, T]) & : & u_{1} \mapsto y_{1}, & y_{1}(k, \cdot)=\Sigma u_{1}(k, \cdot) \\
P_{\Sigma_{1}}: L_{e}^{p}(\mathbb{N} \times[0, T]) \rightarrow L_{e}^{p}(\mathbb{N} \times[0, T]) & : & u_{1} \mapsto y_{1}, & y_{1}(k, \cdot)=\Sigma_{1} u_{1}(k, \cdot)
\end{array}
$$

where $\Sigma$ and $\Sigma_{1}$ are defined in subsection 2.1 .

The controller $C(k, t): L_{e}^{p}(\mathbb{N} \times[0, T]) \rightarrow L_{e}^{p}(\mathbb{N} \times[0, T])$ will represent the map $y_{2} \mapsto u_{2}$ and is required to be causal.

The specific iterative learning controller considered in this paper is defined as follows:

$$
\begin{aligned}
& C_{\theta_{0}}: L_{e}^{p}(\mathbb{N} \times[0, T]) \rightarrow L_{e}^{p}(\mathbb{N} \times[0, T]) \quad: \quad y_{2} \quad \mapsto u_{2}, \\
& u_{2}(k, \cdot)=-\theta_{k} y_{2}(k, \cdot) \\
& \theta_{k}=\theta_{k-1}+\alpha\left\|y_{2}(k-1, \cdot)\right\|_{L^{p}[0, T]}^{p} \\
& \theta_{0} \in(0, \infty), \quad y_{2}(0, \cdot)=0 .
\end{aligned}
$$

The learning gain $\alpha>0$ is taken to be fixed and positive throughout the paper. This controller forms a simple variant on the controllers previously in ILC, for example this is a special case of the ILC design considered in [13] (the feedforward terms have been suppressed) and see also [6] for related designs.

It is simple to observe that the closed loop $\left[P_{\Sigma_{1}}, C_{\theta_{0}}\right]$ is globally well-posed for any LTI plant $\Sigma_{1}$, since it forms a linear system along each trial.

\subsection{Gap metrics and the robust stability margin}

We give two definitions of variants of the gap metric from [7]. Let

$$
\mathcal{O}_{\Sigma_{1}, \Sigma_{2}}^{\mathcal{W}}=\left\{\Phi: \mathcal{G}_{\Sigma_{1}} \rightarrow \mathcal{G}_{\Sigma_{2}} \mid \Phi \text { is causal, surjective, and } \Phi(0)=0\right\} .
$$

where,

$$
\vec{\delta}_{\mathcal{W}}\left(\Sigma_{1}, \Sigma_{2}\right)=\inf _{\Phi \in \mathcal{O}_{\Sigma_{1}, \Sigma_{2}}} \sup _{\substack{x \in \mathcal{G}_{\Sigma_{1}} \backslash\{0\}, \tau \in \mathcal{T} \backslash 0}}\left(\frac{\left\|\left.T_{\tau}(\Phi-I)\right|_{\mathcal{G}_{\Sigma_{1}}} x\right\|_{\mathcal{W}}}{\left\|T_{\tau} x\right\|_{\mathcal{W}}}\right),
$$

This defines a directed gap appropriate for global applications.

Let $B_{r} \subset \mathcal{W}$ denote the ball centred at 0 and of radius $r>0$ in $\mathcal{W}$. Then let

$$
\vec{\delta}_{\mathcal{W}(r)}\left(\Sigma_{1}, \Sigma_{2}\right):=\inf _{\Phi \in \mathcal{O}_{\Sigma_{1}, \Sigma_{2}}} \sup _{\substack{x \in \mathcal{G}_{\Sigma_{1}} \cap B_{r} \backslash\{0\} \\ \tau \in \mathcal{T} \backslash 0}}\left(\frac{\left\|\left.T_{\tau}(\Phi-I)\right|_{\mathcal{G}_{\Sigma_{1}}} x\right\|_{\mathcal{W}}}{\left\|T_{\tau} x\right\|_{\mathcal{W}}}\right)
$$


where

$$
\mathcal{O}_{\Sigma_{1}, \Sigma_{2}}^{\mathcal{W}(r)}:=\left\{\begin{array}{ll}
\Phi: \mathcal{G}_{\Sigma_{1}} \cap B_{r} \rightarrow \mathcal{G}_{\Sigma_{2}} \mid & \begin{array}{l}
\Phi \text { is causal, } \Phi(0)=0 \text { and } \\
T_{\tau}(\Phi-I) T_{\tau} \text { is compact for all } \tau>0
\end{array}
\end{array}\right\} .
$$

This defines a directed gap appropriate for regional applications.

In the case of the signal space $L^{2}\left[\mathbb{R}_{+}\right]$, the gap can be directly related to the classical notion of uncertainty considered in $\mathcal{H}^{\infty}$ control [17],[15] as follows. Suppose $\Sigma_{1}, \Sigma_{2} \in \mathcal{R}$, where $\mathcal{R}$ represents the set of all proper transfer functions. We let $N_{1}, D_{1}, N_{2}, D_{2} \in \mathcal{R} \mathcal{H}^{\infty}$ form normalized coprime factorisatons of $\Sigma_{1}, \Sigma_{2}$ :

$$
\Sigma_{i}=N_{i} D_{i}^{-1}, \quad N_{i}^{*} N_{i}+D_{i}^{*} D_{i}=1, \quad i=1,2 .^{2}
$$

Then the directed gap between $\Sigma_{1}$ and $\Sigma_{2}$ is given by:

$$
\vec{\delta}_{0}\left(\Sigma_{1}, \Sigma_{2}\right):=\inf \left\{\left\|\left(\Delta_{N}, \Delta_{D}\right)\right\|_{\mathcal{H}^{\infty}} \mid \Delta_{N}, \Delta_{D} \in R \mathcal{H}^{\infty}, \Sigma_{2}=\left(N_{1}+\Delta_{N}\right)\left(D_{1}+\Delta_{D}\right)^{-1}\right\},
$$

The $H_{2}$ gap between $\Sigma_{1}$ and $\Sigma_{2}$ is then given by

$$
\delta_{0}\left(\Sigma_{1}, \Sigma_{2}\right) \equiv \delta_{0}\left(\Sigma_{2}, \Sigma_{1}\right)=\max \left\{\vec{\delta}_{0}\left(\Sigma_{1}, \Sigma_{2}\right), \vec{\delta}_{0}\left(\Sigma_{2}, \Sigma_{1}\right)\right\}
$$

and measures the size of the smallest stable co-prime factor difference between normalized coprime factorizations of the two plants. If $\delta_{0}\left(\Sigma_{1}, \Sigma_{2}\right)<1$ then it is shown in [7] that

$$
\vec{\delta}_{L^{2}\left[\mathbb{R}_{+}\right](r)}\left(\Sigma_{1}, \Sigma_{2}\right)=\vec{\delta}_{L^{2}\left[\mathbb{R}_{+}\right]}\left(\Sigma_{1}, \Sigma_{2}\right)=\vec{\delta}_{0}\left(\Sigma_{1}, \Sigma_{2}\right) .
$$

Finally we define the robustness margin at disturbance level $d>0$ as follows:

$$
\begin{aligned}
& B_{P_{\Sigma}, C}(d)=\sup \left\{r \geq 0 \mid \vec{\delta}\left(\Sigma, \Sigma_{1}\right) \leq r \quad \Longrightarrow \quad\left\|\Pi_{P_{\Sigma_{1}}, C}\left(u_{0}, y_{0}\right)\right\|_{L^{p}(\mathbb{N} \times[0, T])}<\infty\right. \\
& \text { for all } \left.\left\|\left(u_{0}, y_{0}\right)\right\|_{L^{p}(\mathbb{N} \times[0, T])} \leq d\right\} \text {. }
\end{aligned}
$$

In the $\mathcal{H}^{2}$, LTI setting, it is known that $B_{P, C}(d)=b_{P, C}=\left\|\Pi_{P, C}\right\|^{-1}$ for all $d>0$. Our aim is to show that the controllers considered in the following section have the property that $B_{P, C}(d)>0$ for all $d>0$, and further to provide explicit bounds for the robustness margins.

\section{Robust Stability Theory for ILC}

\subsection{Gap metrics for ILC}

We now establish relations between the $L^{p}(\mathbb{N} \times[0, T])$ gap between two plants $P_{\Sigma_{1}}, P_{\Sigma_{2}}$ and the $L^{p}\left[\mathbb{R}_{+}\right], L^{p}[0, T]$ gap distances between $\Sigma_{1}$ and $\Sigma_{2}$.

Theorem 3.1 Let $1 \leq p \leq \infty$ and suppose $\Sigma_{1}, \Sigma_{2} \in \mathcal{R}$. Then

$$
\vec{\delta}_{L^{p}(\mathbb{N} \times[0, T])}\left(P_{\Sigma_{1}}, P_{\Sigma_{2}}\right) \leq \vec{\delta}_{L^{p}[0, T]}\left(\Sigma_{1}, \Sigma_{2}\right) \leq \vec{\delta}_{L^{p}\left[\mathbb{R}_{+}\right]}\left(\Sigma_{1}, \Sigma_{2}\right)
$$

and for $r>0$,

$$
\vec{\delta}_{L^{p}(\mathbb{N} \times[0, T])(r)}\left(P_{\Sigma_{1}}, P_{\Sigma_{2}}\right) \leq \vec{\delta}_{L^{p}[0, T](r)}\left(\Sigma_{1}, \Sigma_{2}\right) \leq \vec{\delta}_{L^{p}\left[\mathbb{R}_{+}\right]}\left(\Sigma_{1}, \Sigma_{2}\right) .
$$

\footnotetext{
${ }^{2}$ Here $N^{*}(s):=\overline{N(-\bar{s})}^{T}$
} 
Proof. Let $\Phi \in \mathcal{O}_{\Sigma_{1}, \Sigma_{2}}^{L^{p}[0, T]}$ and define

$$
\begin{aligned}
\Psi & : \quad L^{p}(\mathbb{N} \times[0, T]) \times L^{p}(\mathbb{N} \times[0, T]) \rightarrow L^{p}(\mathbb{N} \times[0, T]) \times L^{p}(\mathbb{N} \times[0, T]) \\
(\Psi w)(k, t) & =(\Phi w(k, \cdot))(t)
\end{aligned}
$$

It is straightforward to observe that $\Psi \in \mathcal{O}_{P_{\Sigma_{1}}, P_{\Sigma_{2}}}^{L^{p}(\mathbb{N} \times[0, T])}$. Then,

$$
\begin{aligned}
& \vec{\delta}_{L^{p}(\mathbb{N} \times[0, T])}\left(P_{\Sigma_{1}}, P_{\Sigma_{2}}\right) \leq \sup _{\substack{w \in \mathcal{G}_{P_{\Sigma_{1}} \backslash\{0\}} \\
t \in \mathbb{N} \times[0, T] \backslash\{0\}}} \frac{\left\|T_{t}(I-\Psi) w\right\|_{L^{p}(\mathbb{N} \times[0, T])}}{\left\|T_{t} w\right\|_{L^{p}(\mathbb{N} \times[0, T])}} \\
& \leq \sup _{\substack{w \in \mathcal{G}_{P_{\Sigma_{1}}} \backslash\{0\} \\
k \geq 1, T \geq \tau>0}} \frac{\sum_{i=1}^{k-1}\|(I-\Phi) w(i, \cdot)\|_{L^{p}[0, T]}+\left\|T_{\tau}(I-\Phi) w(k, \cdot)\right\|_{L^{p}[0, T]}}{\sum_{i=1}^{k-1}\|w(i, \cdot)\|_{L^{p}[0, T]}+\left\|T_{\tau} w(k, \cdot)\right\|_{L^{p}[0, T]}} \\
& \leq \sup _{w \in \mathcal{G}_{P_{\Sigma_{1}}} \backslash\{0\}} \max \left\{\left\|\left.(I-\Phi)\right|_{\mathcal{G}_{\Sigma_{1}}^{L^{p}[0, T]}}\right\|_{L^{p}[0, T]},\left\|\left.T_{\tau}(I-\Phi)\right|_{\mathcal{G}_{\Sigma_{1}}^{L^{p}[0, T]}}\right\|_{L^{p}[0, T]}\right\} \\
& \leq \sup _{T \geq \tau>0}\left\|\left.T_{\tau}(I-\Phi)\right|_{\mathcal{G}_{\Sigma_{1}}^{L^{p}[0, T]}}\right\|_{L^{p}[0, T]} \\
& =\vec{\delta}_{L^{p}[0, T]}\left(\Sigma_{1}, \Sigma_{2}\right)
\end{aligned}
$$

Since $\Phi \in \mathcal{O}_{\Sigma_{1}, \Sigma_{2}}^{L^{p}[0, T]}$ was arbitrary, it follows that

$$
\vec{\delta}_{L^{p}(\mathbb{N} \times[0, T])}\left(P_{\Sigma_{1}}, P_{\Sigma_{2}}\right) \leq \vec{\delta}_{L^{p}[0, T]}\left(\Sigma_{1}, \Sigma_{2}\right)
$$

as required. The inequality $\vec{\delta}_{L^{p}(\mathbb{N} \times[0, T])(r)}\left(P_{\Sigma_{1}}, P_{\Sigma_{2}}\right) \leq \vec{\delta}_{L^{p}[0, T](r)}\left(\Sigma_{1}, \Sigma_{2}\right)$ follows by a similar bound once we have shown that $\Phi \in \mathcal{O}_{\Sigma_{1}, \Sigma_{2}}^{L^{p}[0, T](r)}$ implies $\Psi \in \mathcal{O}_{P_{\Sigma_{1}}, P_{\Sigma_{2}}}^{L^{p}(\mathbb{N} \times[0, T])(r)}$. Note that $\Psi$ is causal and $\Psi(0)=0$ as previously, thus it remains to show that if $T_{\tau}(\Phi-I) T_{\tau}$ is compact for all $\tau>0$, then $T_{(k, t)}(\Psi-I) T_{(k, t)}$ is also compact for all $(k, t) \in \mathbb{N} \times[0, T] \backslash\{0\}$.

Let $(k, t) \in \mathbb{N} \times[0, T] \backslash\{0\} . T_{(k, t)}(\Psi-I) T_{(k, t)}$ is continuous since $T_{(k, t)}(\Psi-I) T_{(k, t)}$ is linear and bounded. Let $\Omega \subset L^{p}(\mathbb{N} \times[0, T]) \times L^{p}(\mathbb{N} \times[0, T])$ be a bounded set and let $\left\{w_{n}\right\}_{n \geq 1}$ be a sequence in $\Omega$. Since $Q_{\tau}=T_{\tau}(\Phi-I) T_{\tau}$ is compact, hence maps bounded sets to relatively compact sets for all $\tau>0$, it follows that there exists a bounded subsequence $\left\{w_{n_{i}}(k, \cdot)\right\}_{i \geq 1} \subset \Omega$ such that $Q_{T} w_{n_{i}}(1, \cdot), \ldots, Q_{T} w_{n_{i}}(k-1, \cdot)$ and $Q_{t} w_{n_{i}}(k, \cdot)$ all converge in $L^{p}[0, T]$ as $i \rightarrow \infty$. Hence since

$$
T_{(k, t)}(\Psi-I) T_{(k, t)} w(\cdot, \cdot)=\left(Q_{T} w(1, \cdot), \ldots, Q_{T} w(k-1, \cdot), Q_{t} w(k, \cdot)\right)
$$

it follows that $T_{(k, t)}(\Psi-I) T_{(k, t)} w_{n_{i}}$ converges in $\overline{T_{(k, t)}(\Psi-I) T_{(k, t)} \Omega}$ as $i \rightarrow \infty$. Hence $T_{(k, t)}(\Psi-$ $I) T_{(k, t)}$ is compact for all $(k, t) \in \mathbb{N} \times[0, T] \backslash\{0\}$, and $\Psi \in \mathcal{O}_{\Sigma_{1}, \Sigma_{2}}^{L^{p}(\mathbb{N} \times[0, T])(r)}$ as required.

We now show that $\vec{\delta}_{L^{p}[0, T]}\left(\Sigma_{1}, \Sigma_{2}\right) \leq \vec{\delta}_{L^{p}\left[\mathbb{R}_{+}\right]}\left(\Sigma_{1}, \Sigma_{2}\right)$. Since $T_{T} \Phi T_{T} \in \mathcal{O}_{\Sigma_{1}, \Sigma_{2}}^{L^{p}[0, T]}$ for all $\Phi \in$ $\mathcal{O}_{\Sigma_{1}, \Sigma_{2}}^{L^{p}\left[\mathbb{R}_{+}\right]}$, and since $\Sigma \in \mathcal{R}$ is stabilizable,

$$
\left.\mathcal{G}_{\Sigma_{1}}^{L^{p}\left[\mathbb{R}_{+}\right]}\right|_{[0, T]}=\mathcal{G}_{\Sigma_{1}}^{L^{p}[0, T]}
$$


it follows that:

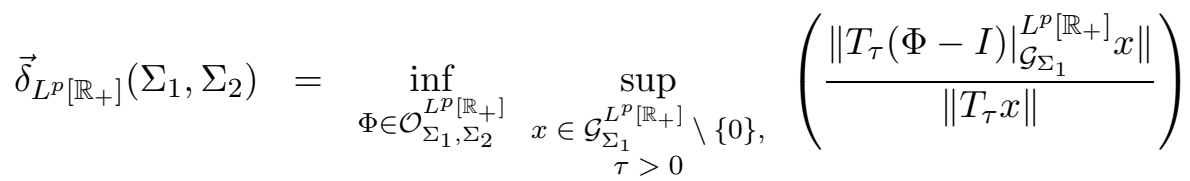

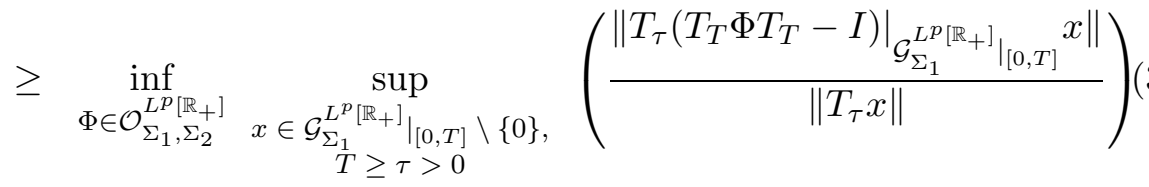

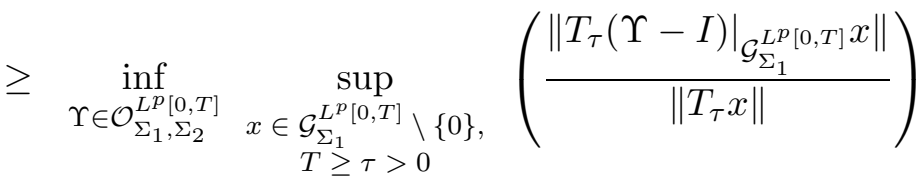

$$
\begin{aligned}
& =\vec{\delta}_{L^{p}[0, T]}\left(\Sigma_{1}, \Sigma_{2}\right)
\end{aligned}
$$

as required. The inequality $\vec{\delta}_{L^{p}[0, T](r)}\left(\Sigma_{1}, \Sigma_{2}\right) \leq \vec{\delta}_{L^{p}\left[\mathbb{R}_{+}\right](r)}\left(\Sigma_{1}, \Sigma_{2}\right)$ follows in a similar manner, since $T_{T} \Phi T_{T} \in \mathcal{O}_{\Sigma_{1}, \Sigma_{2}}^{L^{p}[0, T](r)}$ for all $\Phi \in \mathcal{O}_{\Sigma_{1}, \Sigma_{2}}^{L^{p}\left[\mathbb{R}_{+}\right](r)}$ (since $T_{\tau}(\Phi-I) T_{\tau}=T_{\tau}\left(T_{T} \Phi T_{T}-I\right) T_{\tau}$ for all $T \geq \tau>0$, the compactness of $T_{\tau}\left(T_{T} \Phi T_{T}-I\right) T_{\tau}$ follows from the compactness of $\left.T_{\tau}(\Phi-I) T_{\tau}\right)$.

\subsection{Robust stability theory for ILC}

We now establish the following global robust stability result. It should be noted that the stability margin is determined by the gain of the $2 \mathrm{D}$ closed loop operator $\Pi_{P_{\Sigma}, C}$, whilst the gap is measured by the classical $1 \mathrm{D}$ measure of uncertainty $\vec{\delta}_{L^{p}\left[\mathbb{R}_{+}\right]}\left(\Sigma, \Sigma_{1}\right)$ :

Theorem 3.2 Let $1 \leq p \leq \infty$ and suppose $\Sigma, \Sigma_{1} \in \mathcal{R}, C$ is causal, $C(0)=0,\left[P_{\Sigma}, C\right]$ is stable and $\left[P_{\Sigma_{1}}, C\right]$ is globally well posed. Then

$$
B_{P_{\Sigma}, C}(d) \geq \frac{1}{\left\|\Pi_{P_{\Sigma}, C}\right\|_{L^{p}(\mathbb{N} \times[0, T])}} \quad \text { for all } d \geq 0 .
$$

If $\vec{\delta}_{L^{p}\left[\mathbb{R}_{+}\right]}\left(\Sigma, \Sigma_{1}\right)\left\|\Pi_{P_{\Sigma}, C}\right\|_{L^{p}(\mathbb{N} \times[0, T])}=\epsilon<1$, then:

$$
\left\|\Pi_{P_{\Sigma_{1}}, C}\right\|_{L^{p}(\mathbb{N} \times[0, T])} \leq\left\|\Pi_{P_{\Sigma}, C}\right\|_{L^{p}(\mathbb{N} \times[0, T])}\left(\frac{1+\vec{\delta}_{L^{p}\left[\mathbb{R}_{+}\right]}\left(\Sigma, \Sigma_{1}\right)}{1-\epsilon}\right) .
$$

Proof. Let $\Upsilon: L^{p}(\mathbb{N} \times[0, T]) \rightarrow L^{p}\left[\mathbb{R}_{+}\right]$denote the isomorphism $(\Upsilon u)(k, s)=u((k-1) T+s)$. Define

$$
\begin{array}{rlrl}
P: L_{e}^{p}\left[\mathbb{R}_{+}\right] \rightarrow L_{e}^{p}\left[\mathbb{R}_{+}\right] & P u=\left(\Upsilon \circ P_{\Sigma} \circ \Upsilon^{-1}\right) u, \\
P_{1}: L_{e}^{p}\left[\mathbb{R}_{+}\right] \rightarrow L_{e}^{p}\left[\mathbb{R}_{+}\right] & & P_{1} u=\left(\Upsilon \circ P_{\Sigma_{1}} \circ \Upsilon^{-1}\right) u, \\
K: L_{e}^{p}\left[\mathbb{R}_{+}\right] \rightarrow L_{e}^{p}\left[\mathbb{R}_{+}\right] & & K u=\left(\Upsilon \circ C \circ \Upsilon^{-1}\right) u .
\end{array}
$$

Since $\Upsilon$ is isometric, and by Theorem 3.1, we have

$$
\vec{\delta}_{L^{p}\left[\mathbb{R}_{+}\right]}\left(P, P_{1}\right)=\vec{\delta}_{L^{p}(\mathbb{N} \times[0, T])}\left(P_{\Sigma}, P_{\Sigma_{1}}\right) \leq \vec{\delta}_{L^{p}\left[\mathbb{R}_{+}\right]}\left(\Sigma, \Sigma_{1}\right) .
$$


Noting that $P, P_{1}, K$ are causal and satisfy $P(0)=P_{1}(0)=K(0)=0,[7$, Theorem 1$]$ then establishes:

$$
\left\|\Pi_{P_{1}, K}\right\|_{L^{p}\left[\mathbb{R}_{+}\right]} \leq\left\|\Pi_{P, K}\right\|_{L^{p}\left[\mathbb{R}_{+}\right]}\left(\frac{1+\vec{\delta}_{L^{p}\left[\mathbb{R}_{+}\right]}\left(P, P_{1}\right)}{1-\vec{\delta}_{L^{p}\left[\mathbb{R}_{+}\right]}\left(P, P_{1}\right)\left\|\Pi_{P, K}\right\|_{\left[\mathbb{R}_{+}\right]}}\right) .
$$

Since $\Upsilon$ is isometric, we also have:

$$
\begin{aligned}
\left\|\Pi_{P, K}\right\|_{L^{p}\left[\mathbb{R}_{+}\right]} & =\left\|\Pi_{P_{\Sigma}, C}\right\|_{L^{p}(\mathbb{N} \times[0, T])} \\
\left\|\Pi_{P_{1}, K}\right\|_{L^{p}\left[\mathbb{R}_{+}\right]} & =\left\|\Pi_{P_{\Sigma_{1}}, C}\right\|_{L^{p}(\mathbb{N} \times[0, T])}
\end{aligned}
$$

Inequality (3.32) now follows from inequality (3.35) by (3.34) and (3.36) as required.

The specific application to the adaptive ILC algorithm considered in this paper requires a further robust stability result which is regional in nature:

Theorem 3.3 Let $1 \leq p \leq \infty$ and suppose $\Sigma, \Sigma_{1} \in \mathcal{R}, C$ is causal, $C(0)=0,\left[P_{\Sigma}, C\right]$ is $B_{r}$-stable and $\left[P_{\Sigma_{1}}, C\right]$ is globally well posed. Let $0 \leq \epsilon<1$. Then

$$
B_{P_{\Sigma}, C}(r(1-\epsilon)) \geq \frac{\epsilon}{\left\|\left.\Pi_{P_{\Sigma}, C}\right|_{B_{r}}\right\|_{L^{p}(\mathbb{N} \times[0, T])}} .
$$

If $\vec{\delta}_{L^{p}\left[\mathbb{R}_{+}\right]}\left(\Sigma, \Sigma_{1}\right)\left\|\left.\Pi_{P_{\Sigma}, C}\right|_{B_{r}}\right\|_{L^{p}(\mathbb{N} \times[0, T])} \leq \epsilon$, then:

$$
\left\|\left.\Pi_{P_{\Sigma_{1}}, C}\right|_{B_{r(1-\epsilon)}}\right\|_{L^{p}(\mathbb{N} \times[0, T])} \leq\left\|\left.\Pi_{P_{\Sigma}, C}\right|_{B_{r}}\right\|_{L^{p}(\mathbb{N} \times[0, T])}\left(\frac{1+\vec{\delta}_{L^{p}\left[\mathbb{R}_{+}\right]}\left(\Sigma, \Sigma_{1}\right)}{1-\epsilon}\right) .
$$

Proof. The proof establishes the result from Theorem 3.1 and [7, Theorem 4] analogously to the deduction of Theorem 3.2 from Theorem 3.1 and [7, Theorem 1].

\section{Proof of the $L^{2}$ result}

Let $p=2$, so $\mathcal{U}=\mathcal{Y}=L^{2}(\mathbb{N} \times[0, T])$. We first establish an intermediate result for a first order system. In the final analysis, this result will play the role of providing bounds on the internal signals from the trial index at which the closed loop has achieved a certain 1D stable pole location in the dynamics along the trial.

Proposition 4.1 Let $M>0, \alpha>0, \beta>0$ and suppose $a_{11}+\beta>M$. Consider the system:

$$
\begin{aligned}
\frac{\partial}{\partial t} y_{1}(k, t) & =-a_{11} y_{1}(k, t)+u_{1}(k, t)+v(k, t) \\
u_{2}(k, t) & =-\theta_{k} y_{2}(k, t) \\
\theta_{k} & =\beta+\alpha \sum_{i=0}^{k-1}\left\|y_{2}(i, \cdot)\right\|_{L^{2}[0, T]}^{2} \\
y_{1}(k, 0) & =0, \quad \text { for all } k \in \mathbb{N}, \\
y_{2}(0, t) & =0, \quad \text { for all } t \in[0, T],
\end{aligned}
$$


where $\|v(i, \cdot)\|_{L^{2}[0, T]} \leq M\left\|y_{1}(i, \cdot)\right\|_{L^{2}[0, T]}, i \geq 1$.

Then

$$
\left\|\left(\begin{array}{l}
u_{1} \\
y_{1}
\end{array}\right)\right\|_{\mathcal{U} \times \mathcal{Y}} \leq \nu\left(\beta, \epsilon,\left\|\left(\begin{array}{l}
u_{0} \\
y_{0}
\end{array}\right)\right\|_{\mathcal{U} \times \mathcal{Y}}\right)
$$

where $\epsilon=a_{11}+\beta-M$ and

$$
\nu(\beta, \epsilon, r)=\left(\gamma_{1} r^{2}+3\left(1+\frac{\gamma_{2} r^{2}}{\epsilon}\right)^{2} r^{2}+3\left(2+\frac{\beta}{\epsilon}\right)^{2} \gamma_{2}^{2} r^{6}\right)^{\frac{1}{2}}
$$

and

$$
\begin{aligned}
\gamma_{1} & =\frac{3}{\epsilon^{2}}+3\left(1+\frac{\beta}{\epsilon}\right)^{2} \\
\gamma_{2} & =\beta+3 \alpha\left(1+3\left(1+\frac{\beta}{\epsilon}\right)^{2}\right)+\frac{9 \alpha}{\epsilon^{2}}
\end{aligned}
$$

Proof. Firstly we note that:

$$
\begin{aligned}
\frac{\partial}{\partial t} y_{1}(k, t) & =-a_{11} y(k, t)+u_{0}(k, t)-u_{2}(k, t)+v(k, t) \\
& =-a_{11} y_{1}(k, t)+u_{0}(k, t)+\theta_{k}\left(y_{0}(k, t)-y_{1}(k, t)\right)+v(k, t) \\
& =-\left(a_{11}+\theta_{k}\right) y_{1}(k, t)+u_{0}(k, t)+\theta_{k} y_{0}(k, t)+v(k, t)
\end{aligned}
$$

Let $V: \mathbb{R} \rightarrow \mathbb{R}_{+}$be defined as $V\left(y_{1}\right)=\frac{1}{2} y_{1}^{2}$. Then

$$
\frac{\partial}{\partial t} V=y_{1}(k, t) \frac{\partial}{\partial t} y_{1}(k, t)=-\left(a_{11}+\theta_{k}\right) y_{1}^{2}(k, t)+y_{1}(k, t)\left(u_{0}(k, t)+\theta_{k} y_{0}(k, t)+v(k, t)\right) .
$$

Integrating on $[0, T]$, and using the inequality $\|v(k, \cdot)\| \leq M\left\|y_{1}(k, \cdot)\right\|$ we obtain:

$$
\begin{aligned}
\frac{1}{2} y_{1}^{2}(k, T) \leq & -\left(a_{11}+\theta_{k}\right)\left\|y_{1}(k, \cdot)\right\|_{L^{2}[0, T]}^{2} \\
& +\left\|y_{1}(k, \cdot)\right\|_{L^{2}[0, T]}\left(\left\|u_{0}(k, \cdot)\right\|_{L^{2}[0, T]}+\theta_{k}\left\|y_{0}(k, \cdot)\right\|_{L^{2}[0, T]}+\|v(k, \cdot)\|_{L^{2}[0, T]}\right) \\
\leq & -\left(a_{11}+\theta_{k}-M\right)\left\|y_{1}(k, \cdot)\right\|_{L^{2}[0, T]}^{2} \\
& +\left\|y_{1}(k, \cdot)\right\|_{L^{2}[0, T]}\left(\left\|u_{0}(k, \cdot)\right\|_{L^{2}[0, T]}+\theta_{k}\left\|y_{0}(k, \cdot)\right\|_{L^{2}[0, T]}\right) .
\end{aligned}
$$

Hence,

$$
\begin{aligned}
\left\|y_{1}(k, \cdot)\right\|_{L^{2}[0, T]}^{2} & \leq\left(\frac{1}{a_{11}+\theta_{k}-M}\left(\left\|u_{0}(k, \cdot)\right\|_{L^{2}[0, T]}+\theta_{k}\left\|y_{0}(k, \cdot)\right\|_{L^{2}[0, T]}\right)\right)^{2} \\
& \leq 3 \frac{1}{\left(a_{11}+\beta-M\right)^{2}}\left\|u_{0}(k, \cdot)\right\|_{L^{2}[0, T]}^{2}+3\left(1+\frac{\beta}{a_{11}+\beta-M}\right)^{2}\left\|y_{0}(k, \cdot)\right\|_{L^{2}[0, T]}^{2} .
\end{aligned}
$$

By inequality (4.47) we obtain:

$$
\left\|y_{1}\right\|_{\mathcal{Y}}^{2} \leq \frac{3}{\left(a_{11}+\beta-M\right)^{2}}\left\|u_{0}\right\|_{\mathcal{U}}^{2}+3\left(1+\frac{\beta}{a_{11}+\beta-M}\right)^{2}\left\|y_{0}\right\|_{\mathcal{Y}}^{2},
$$


SO

$$
\left\|y_{1}\right\|_{\mathcal{Y}}^{2} \leq \gamma_{1}\left\|\left(u_{0}, y_{0}\right)^{T}\right\|_{\mathcal{U} \times \mathcal{Y}}^{2} .
$$

To bound $\left\|u_{1}\right\|_{\mathcal{U}}$, we first observe that:

$$
\begin{aligned}
\theta_{k}=\beta+\alpha \sum_{i=1}^{k-1}\left\|y_{2}(i, \cdot)\right\|_{L^{2}[0, T] \leq}^{2} \leq & +3 \alpha \sum_{i=1}^{k-1}\left(\left(1+3\left(1+\frac{\beta}{a_{11}+\beta-M}\right)^{2}\right)\left\|y_{0}(i, \cdot)\right\|_{L^{2}[0, T]}^{2}\right. \\
& \left.+\frac{3}{\left(a_{11}+\beta-M\right)^{2}}\left\|u_{0}(i, \cdot)\right\|_{L^{2}[0, T]}^{2}\right) \\
\leq & \beta+3 \alpha\left(1+3\left(1+\frac{\beta}{a_{11}+\beta-M}\right)^{2}\right)\left\|y_{0}\right\|_{\mathcal{Y}}^{2} \\
& +\frac{9 \alpha}{\left(a_{11}+\beta-M\right)^{2}}\left\|u_{0}\right\|_{\mathcal{U}}^{2} \\
= & \gamma_{2}\left\|\left(u_{0}, y_{0}\right)^{T}\right\|_{\mathcal{U} \times \mathcal{Y}}^{2} .
\end{aligned}
$$

Then,

$$
\begin{aligned}
\left\|u_{1}(k, \cdot)\right\|_{L^{2}[0, T]} \leq & \left\|u_{0}(k, \cdot)\right\|_{L^{2}[0, T]}+\theta_{k}\left\|y_{2}(k, \cdot)\right\|_{L^{2}[0, T]} \\
\leq & \left\|u_{0}(k, \cdot)\right\|_{L^{2}[0, T]}+\theta_{k}\left\|y_{0}(k, \cdot)\right\|_{L^{2}[0, T]}+\theta_{k}\left\|y_{1}(k, \cdot)\right\|_{L^{2}[0, T]} \\
\leq & \left(1+\frac{\theta_{k}}{a_{11}+\beta-M}\right)\left\|u_{0}(k, \cdot)\right\|_{L^{2}[0, T]} \\
& +\theta_{k}\left(2+\frac{\beta}{a_{11}+\beta-M}\right)\left\|y_{0}(k, \cdot)\right\|_{L^{2}[0, T]} .
\end{aligned}
$$

By inequality (4.51) we see that

$$
\begin{aligned}
\left\|u_{1}\right\|_{\mathcal{U}}^{2}= & \sum_{k=1}^{\infty}\left\|u_{1}(k, \cdot)\right\|_{L^{2}[0, T]}^{2} \\
\leq & 3 \sum_{k=1}^{\infty}\left(\left(1+\frac{\theta_{k}}{a_{11}+\beta-M}\right)^{2}\left\|u_{0}(k, \cdot)\right\|_{L^{2}[0, T]}^{2}\right. \\
& \left.+\theta_{k}^{2}\left(2+\frac{\beta}{a_{11}+\beta-M}\right)^{2}\left\|y_{0}(k, \cdot)\right\|_{L^{2}[0, T]}^{2}\right) \\
\leq & 3\left(1+\frac{\sup _{k \geq 1} \theta_{k}}{a_{11}+\beta-M}\right)^{2}\left\|u_{0}\right\|_{\mathcal{U}}^{2}+3\left(2+\frac{\beta}{a_{11}+\beta-M}\right)^{2}\left(\sup _{k \geq 1} \theta_{k}\right)^{2}\left\|y_{0}\right\|_{\mathcal{Y}}^{2},
\end{aligned}
$$

so if $\left\|\left(u_{0}, y_{0}\right)^{T}\right\|_{\mathcal{U} \times \mathcal{Y}} \leq r$ then

$$
\left\|u_{1}\right\|_{\mathcal{U}}^{2} \leq 3\left(1+\frac{\gamma_{2} r^{2}}{a_{11}+\beta-M}\right)^{2} r^{2}+3\left(2+\frac{\beta}{a_{11}+\beta-M}\right)^{2} \gamma_{2}^{2} r^{6}
$$

and the result follows. 
We now give the main result, which establishes a quadratic lower bound on the robust stability margin.

Theorem 4.2 Let the plant $P_{\Sigma}$ and the controller $C_{0}$ be defined by equations $(2.9),(2.11)$ where $\Sigma \in \mathcal{M}_{M}^{1}$. Then for all $\epsilon>0$

$$
B_{P_{\Sigma}, C}(r) \geq \min \left\{\frac{r}{\left.\nu\left(\epsilon+M-a_{11}+3 \alpha r^{2}(2 \gamma+1)^{2}+1\right), \epsilon, 2 r\right)}, \frac{1}{2 \gamma}\right\},
$$

where $\nu$ is defined as in Proposition 4.1 and

$$
\gamma=\sup _{0 \leq \eta \leq \epsilon+M-a_{11}}\left\|\Pi_{\Sigma, \eta}\right\|_{L^{2}[0, T]} .
$$

Proof. Let $r>0$, and suppose $w_{0}=\left(u_{0}, y_{0}\right)^{T},\left\|w_{0}\right\| \leq r$. Suppose $\Sigma_{1}$ is such that

$$
\vec{\delta}_{L^{2}\left[\mathbb{R}_{+}\right]}\left(\Sigma, \Sigma_{1}\right) \leq \min \left\{\frac{r}{\left.\nu\left(\epsilon+M-a_{11}+3 \alpha r^{2}(2 \gamma+1)^{2}+1\right), \epsilon, 2 r\right)}, \frac{1}{2 \gamma}\right\} .
$$

Consider $\left[P_{\Sigma_{1}}, C_{0}\right]$. Let $K \in[1, \infty]$ be the smallest integer such that

$$
\theta_{K} \geq M-a_{11}+\epsilon
$$

if the above inequality is satisfied and let $K=\infty$ if not. Then by the well posedness of $\left[P_{\Sigma_{1}}, C_{0}\right]$, we have $\theta_{K}<\infty$ and:

$$
\begin{aligned}
\left\|T_{K, 0} y_{2}\right\|^{2} & =\sum_{k=1}^{K-1}\left\|y_{2}(k, \cdot)\right\|_{L^{2}[0, T]}^{2}=\frac{1}{\alpha} \sup _{1 \leq k \leq K} \theta_{k}=\frac{\theta_{K}}{\alpha}, \\
\left\|T_{K, 0} u_{2}\right\|^{2} & =\sum_{k=1}^{K-1}\left\|\theta_{k} y_{2}(k, \cdot)\right\|_{L^{2}[0, T]}^{2} \leq \sum_{k=1}^{K-1}\left\|\theta_{k}\right\|_{L^{\infty}[0, T]}^{2}\left\|y_{2}(k, \cdot)\right\|_{L^{2}[0, T]}^{2} \leq \frac{\theta_{K}^{3}}{\alpha} .
\end{aligned}
$$

and so,

$$
\left\|T_{K, 0} \Pi_{P_{\Sigma_{1}}, C_{0}} w_{0}\right\|^{2} \leq 3\left(\frac{1}{\alpha}\left(\theta_{K}^{3}+\theta_{K}\right)+r^{2}\right)<\infty .
$$

Thus if $K=\infty$ we are done.

On the other hand, suppose $K<\infty$. By shifting the trial index, we obtain

$$
\begin{aligned}
\left\|\Pi_{P_{\Sigma_{1}}, C_{0}} w_{0}\right\| & =\left\|T_{K, 0} \Pi_{P_{\Sigma_{1}}, C_{0}} w_{0}\right\|+\left\|\left(I-T_{K, 0}\right) \Pi_{P_{\Sigma_{1}}, C_{0}} w_{0}\right\| \\
& =\left\|T_{K, 0} \Pi_{P_{\Sigma_{1}}, C_{0}} w_{0}\right\|+\left\|\Pi_{P_{\Sigma_{1}}, C_{\theta_{K}}} v_{0}\right\|,
\end{aligned}
$$

where $v_{0}(k, t)=w_{0}(k-K, t),(k, t) \in \mathbb{N} \times[0, T]$, and thus it suffices to bound $\left\|\Pi_{P_{\Sigma_{1}}, C_{\theta_{K}}} v_{0}\right\|$. Hence consider $\left[P_{\Sigma}, C_{\theta_{K}}\right]$. Note that $\left[P_{\Sigma_{1}}, C_{\theta_{K}}\right]$ is globally well-posed. We first bound $\theta_{K}$. Since by Theorem 3.1, $\vec{\delta}_{L^{2}[0, T]}\left(\Sigma, \Sigma_{1}\right) \leq \vec{\delta}_{L^{2}\left[\mathbb{R}_{+}\right]}\left(\Sigma, \Sigma_{1}\right) \leq \frac{1}{2 \gamma}$, it follows from [7, Theorem 1] that

$$
\left\|\Pi_{\Sigma_{1}, \theta_{K-1}}\right\|_{L^{2}[0, T]} \leq\left\|\Pi_{\Sigma, \theta_{K-1}}\right\|_{L^{2}[0, T]}\left(\frac{1+\vec{\delta}_{L^{2}[0, T]}\left(\Sigma, \Sigma_{1}\right)}{1-\vec{\delta}_{L^{2}[0, T]}\left(\Sigma, \Sigma_{1}\right)\left\|\Pi_{\Sigma, \theta_{K-1}}\right\|_{L^{2}[0, T]}}\right) \leq 2 \gamma+1
$$


Hence

$$
\begin{aligned}
\theta_{K} & =\theta_{K-1}+\alpha\left\|y_{2}(k-1, \cdot)\right\|_{L^{2}[0, T]}^{2} \\
& \leq \epsilon+M-a_{11}+3 \alpha r^{2}\left(\left\|(01) \Pi_{\Sigma_{1}, \theta_{K-1}}\right\|_{L^{2}[0, T]}^{2}+1\right) \\
& \leq \epsilon+M-a_{11}+3 \alpha r^{2}\left((2 \gamma+1)^{2}+1\right)
\end{aligned}
$$

By Proposition 4.1, we see that

$$
\left\|\left.\Pi_{P_{\Sigma}, C_{\theta_{K}}}\right|_{B_{2 r}}\right\| \leq \frac{\nu(\beta, \epsilon, 2 r)}{2 r}
$$

where $\nu$ is defined by equation (4.41) with $\left.\beta=\epsilon+M-a_{11}+3 \alpha r^{2}(2 \gamma+1)^{2}+1\right)$. By inequality (4.61), it follows that

$$
\vec{\delta}_{L^{2}\left[\mathbb{R}_{+}\right]}\left(\Sigma, \Sigma_{1}\right)<\frac{1}{2\left\|\left.\Pi_{P_{\Sigma}, C_{\theta_{K}}}\right|_{B_{2 r}}\right\|} .
$$

Hence by Theorem 3.3, with $\epsilon=\frac{1}{2}$, if follows that $\left\|\Pi_{P_{\Sigma_{1}}, C_{\theta_{K}}} \mid B_{r}\right\|<\infty$ and in particular $\left\|\Pi_{P_{\Sigma_{1}}, C_{\theta_{K}}} v_{0}\right\|<\infty$ since $\left\|v_{0}\right\| \leq\left\|w_{0}\right\| \leq r$. This completes the proof.

\section{Simulation Example}

As an example we consider the nominal plant to be an integrator:

$$
\Sigma=\frac{1}{s}
$$

and the true plant to be the integrator with a multiplicative all pass factor perturbation:

$$
\Sigma_{1}^{N}=\frac{N-s}{N+s} \cdot \frac{1}{s}
$$

Straightforward estimations, see $[4,5]$, show

$$
\vec{\delta}_{0}\left(\Sigma, \Sigma_{1}^{N}\right) \rightarrow 0 \quad \text { as } N \rightarrow \infty .
$$

Furthermore, note that there is an $180^{\circ}$ phase difference between the two plants at high frequencies, thus taking this example beyond the previous ILC robustness theory [8] as discussed in the Introduction.

Simulations of the closed loop system with the IlC controller with a learning gain given by $\alpha=0.1$, a trial length of $T=2$ and input and output disturbances given by

$$
u_{0}(k, t)=0, \quad y_{0}(k, t)=\frac{20}{k^{2}} \exp \left(-20(t-1)^{2}\right), \quad(k, t) \in \mathbb{N} \times[0,2],
$$

are shown in figure 2, where the logarithm of the square of the $L^{2}[0,2]$ output error is plotted against the trial number (for the first 25 trials) for integer values of $N, 5 \leq N \leq 30$. This clearly illustrates instability for small values of $N(5 \leq N \leq 11$, whereby the unmodelled dynamics is significant), and long term stability for larger values of $N$, as predicted qualitatively by the theory. 


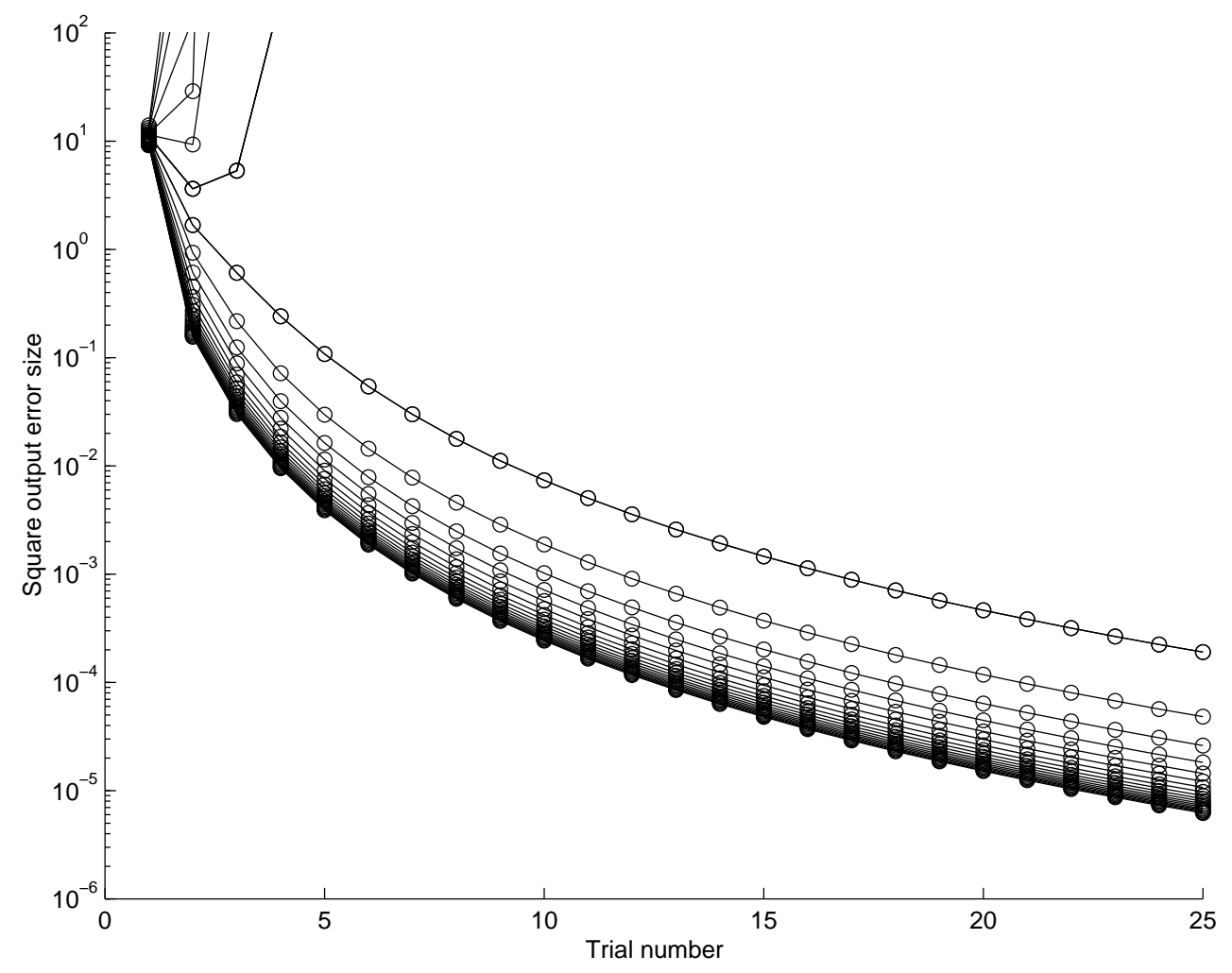

Figure 2: $L^{2}[0, T]$ output error evolution against ILC trial index for $5 \leq N \leq 30$. 


\section{Discussion and Conclusion}

The results in this paper demonstrate that explicit robustness guarantees for a class of ILC designs can be given. The uncertainty classes considered are of a classical type, thus the designs have the same (qualitative) robustness certificates as are obtained by $\mathcal{H}^{\infty}$ controllers. We observe that such classical robustness guarantees have, to date remained elusive in the literature for example, the previous result [8], required the (multiplicative) unmodelled dynamics to have a maximum of $\pm 90^{\circ}$ phase deviations from the nominal model over the entire frequency spectrum, and this is too restrictive to capture many seemingly mild forms of unmodelled dynamics encountered in any application. The results presented are also interpretable 'in the frequency domain', and the example considered in detail is representative of the class of system which lies outside the scope of [8]; note however, that in contrast the results presented here, [8] establishes monotone convergence. More generally the gap or co-prime factor uncertainty model adopted is more general than the other previously considered results in ILC which are restricted to uncertainty models of a multiplicative type, e.g. [10], [14].

In contrast to the situation for LTI plants and controllers, the robustness margins are dependent on the disturbance level: this appears to be a feature of 'learning' systems, see for example the recent related results in adaptive control $[4,5]$ where the margin also is of this form.

The results are limited to a specialized class of ILC design, namely those of an adaptive nature, and are restricted to tracking of the zero trajectory in the presence of disturbances. It is of interest to determine whether the approach can be extended to encompass other approaches to ILC design, to tracking problems and in alternative signal space settings. We have not attempted to consider the optimization of the resulting margins; the problem of optimizing robustness margins and/or performance in ILC is clearly an important area for future consideration, this will involve obtaining tight upper and lower bounds on the margins. We have defined the margin in terms of the 1D gap measure of plant uncertainty. Further work on the interpretation of the 2D gap is clearly important, especially in the cases of short trial lengths when the 1D gap may be unduly conservative.

We consider the results in this paper to be a significant step towards realizing a useful treatment of robustness in the iterative learning context.

\section{Acknowledgement}

The author would like to thank R. Bradley and the reviewers for a number of useful comments on the draft manuscript.

\section{References}

[1] Kawamura S. Arimoto, S. and F. Miyazaki. Bettering operation of robots by learning. J. Robot. Systems, 1:123-140, 1984.

[2] Lewin P. L. Barton, A. D. and D. J. Brown. Practical implementation of a real-time iterative learning position controller. Int. J. Control, 73(10):992-999, 2000. 
[3] Longman R.W. Phan M.Q. Juang J-N. Elci, H. and R. Ugoletti. Simple learning control made practical by zero-phase filtering: Applications to robotics. IEEE Trans. on Circuits and Systems I: Fundamental Theory and Applications, 49(6):753-767, 2002.

[4] M. French. Adaptive control and robustness in the gap metric. IEEE Trans. Autom. Control. To appear.

[5] M. French, A. Ilchmann, and E.P. Ryan. Robustness in the graph topology of a common adaptive controller. SIAM J. Control Optim., 45(5):1736-1757, 2006.

[6] M. French and E. Rogers. Non-linear iterative learning by an adaptive Lyapunov method. Int. J. Control, 73(10):840-850, 2000.

[7] T.T. Georgiou and M.C. Smith. Robustness analysis of nonlinear feedback systems. IEEE Trans. Autom. Control, 42(9):1200-1229, 1997.

[8] T.J. Harte, J. Hätönen, and D.H. Owens. Discrete-time inverse model-based iterative learning control: stability, monotonicity and robustness. Int. J. Control, 78(8):577-586, 2005.

[9] R.W. Longman. Iterative learning control and repetitive control for engineering practice. Int. J. Control, 73(10):930-954, 2000.

[10] Doh T-Y. Moon, J-H. and M.J. Chung. A robust approach to iterative learning control design for uncertain systems. Automatica, 34(8):1001-1004, 1998.

[11] K. Moore. Iterative learning control - an expository overview. Applied and Computational Controls, Signal Processing and Circuits, 1:425-488, 1999.

[12] D.H. Owens and J.J. Hatonen. Iterative learning control - an optimization paradigm. IFAC Annual Reviews in Control, 29:57-60, 2005.

[13] D.H. Owens and G. Munde. Error convergence in an adaptive iterative learning controller. Int. J. Control, 73(10):851-857, 2000.

[14] A. Tayebi and M.B. Zaremba. Robust iterative learning control design is staraightforward for uncertain LTI systems satisfying the robust performance condition. IEEE Trans. Autom. Control, 48(1):101-106, 2003.

[15] G. Vinnicombe. Uncertainty and Feedback: $\mathcal{H}_{\infty}$-shaping Control System Synthesis. Imperial College Press, London, 2001.

[16] G. Zames and A. K. El-Sakkary. Unstable systems and feedback: The gap metric. In Proc. of the Allerton Conf., pages 380-385, 1980.

[17] K. Zhou, J.C. Doyle, and K. Glover. Robust and Optimal Control. Prentice Hall, New Jersey, 1st edition, 1996. 